\title{
Mechanism of Sanren Decoction in Treating Constipation Based on Network Pharmacology
}

\author{
Zihua Guo ${ }^{1, *}$, Yaping Cui ${ }^{1}$, Chuan chuan $\mathrm{Bi}^{2}$, Xia Xiao ${ }^{2}$, Hua Jiang ${ }^{2}$ \\ ${ }^{1}$ Shaanxi University of Traditional Chinese Medicine, Xianyang, Shaanxi 712046, China. \\ ${ }^{2}$ Affiliated Hospital of Shaanxi University of Traditional Chinese Medicine, Xianyang, Shaanxi 712000, China.
}

How to cite this paper: Zihua Guo, Yaping Cui, Chuan chuan Bi, Xia Xiao, Hua Jiang. (2020) Mechanism of Sanren Decoction in Treating Constipation Based on Network Pharmacology. International Journal of Clinical and Experimental Medicine Research, 4(4), 175-181. DOI: 10.26855/ijcemr.2020.10.009

Received: June 17, 2020

Accepted: July 25, 2020

Published: September 10, 2020

*Corresponding author: Zihua Guo, Shaanxi University of Traditional Chinese Medicine, Xianyang, Shaanxi 712046, China.

Email: 1325674248@qq.com

\begin{abstract}
Objective: To analyze the potential mechanism of Sanren Decoction in the treatment of constipation by using network pharmacological methods, and to provide a theoretical reference for further experimental research. Methods: The TCMSP, Uniprot, and Genecards databases were searched, and the targets of Sanren Tang chemical constituents and constipation-related targets were screened. Cytoscape software was used to construct the chemical composition-target-disease network of Sanren Tang. The biological function and signal pathway were analyzed to explore the mechanism of Sanren Decoction in the treatment of constipation. Results: According to the screening conditions [oral bioavailability (OB) $\geq 30$, drug-like (DL) $\geq 0.18$ )], a total of 502 active ingredients and 219 potential drug targets were obtained. A total of 2,842 disease targets were collected in the Genecards database and the UniProt database with "constipation" as the search condition 119 core target proteins of Sanren Decoction for constipation were screened, which mainly involved DRD1, CHRM3, CHRM1, PTGS2, RXRA and so on. The main biological processes of GO include: ubiquitin-like protein ligase binding, kinase-regulated activity, cytokine receptor binding, ubiquitin-protein ligase binding, and protein kinase regulator activity. KEGG enrichment results mainly include: PI3K-Akt signaling pathway, hepatitis B pathway, human cytomegalovirus infection pathway, hepatitis C pathway, Kaposi's sarcoma-associated herpes virus infection pathway, and so on. Conclusion: The mechanism of Sanren Decoction in the treatment of constipation may be related to its multiple components acting on multiple targets, affecting multiple signal pathways related to constipation, and regulating complex biological processes.
\end{abstract}

\section{Keywords}

Sanren Decoction, constipation, network pharmacology, mechanism of action, signal pathway

\section{Introduction}

Constipation is a common clinical digestive tract symptoms, is the defecation difficulty and defecation times to reduce the state of summary. Normal food into the gastrointestinal tract, into digestion and absorption, the residue into feces out of the body, need 24-48 h, if defecation interval more than $48 \mathrm{~h}$, can be regarded as constipation. As a result, when the feces remain in the intestinal cavity for too long, the water is absorbed in excess, the feces become dry, hard, difficult to discharge, then it is manifested as lack of stool meaning or even long time no stool, 
defecation times decreased, < 3 times per week, or frequent stool, but difficult to discharge, accompanied by anal obstruction and defecation feeling [1]. Sanren Tang is first contained in the Qing Dynasty Wu Jutong's "febrile disease on the Jiao section” article 43: headache and cold, body weight pain, tongue white not thirsty, pulse string thin and moistening, pale yellow complexion, chest tightness not hunger, afternoon body heat, if Yin deficiency, disease is difficult to quickly already, known as wet temperature. Sanjen soup master. This prescription is composed of 8 kinds of drugs, such as bitter almond, white cardamom kernel, Coix seed, Magnolia officinalis, Pinellia ternata, flying talc, Baotong grass and light bamboo leaves [2]. Sanren Decoction has a good clinical effect on damp-heat stagnation constipation [3-6], but it is good for dampness-heat stagnation constipation. The mechanism of its action has not been completely clear, the previous study of Sanren decoction in the treatment of constipation only from a certain angle, lack of systematic research. Network pharmacology is to systematically and comprehensively observe the intervention and influence of drugs on the disease network by constructing a multi-level network between "disease-gene-target-drug" and analyzing the relationship between drugs and specific nodes in the network [7]. Therefore, this study combined network pharmacology research ideas with traditional Chinese medicine compound Sanren decoction to explain its therapeutic active components and possible molecular mechanisms in the treatment of constipation, in order to provide a certain basis for subsequent experimental research.

\section{Material methods and results}

\subsection{Chemical Composition and Target Network of San Ren Tang}

Through the TCMSP database [8] (TCMSP, http://tcmspw.com/tcmsp.php), the whole prescription of Sanren Tang was retrieved, and the $\mathrm{OB} \geq 30 \%$ and $0.18 \%$ were used as screening conditions [9]. A total of 502 active chemical constituents were obtained, including 113 bitter almonds, 38 coriander, 71 white nutmeg, 139 Magnolia officinalis, 116 Pinellia ternata, 32 Baitong grass, 0 talc, and 0 bamboo leaves (so no chemicals were removed). Retrieve the target of candidate chemical components through TCMSP database, and the number of target imported proteins collected according tolibrary (UniProtDataba, http: //www.uniport.org/) [10], the chemical composition-action target data set was established by entering the target name and defining the species as human, removing non-human targets, chemical components without targets and repetitive chemical components. A total of 219 action targets corresponding to 502 chemical components were retrieved. Using Cytoscape software association function (Merge) to construct chemical composition-action-target network (Figure 1A), where nodes represent chemical composition and action target and edges represent chemical composition and the interaction relationship between the target of action. From Figure 1B, we can see that there are many chemical components in blindly medicine, the same chemical component can correspond to multiple targets, and the same action target can also correspond to multiple chemical components, which reflect the characteristics of multi-component and multi-target synergistic action of Sanren decoction.

\subsection{Collection and screening of targets related to constipation}

The key words "were jointly retrieved by Genecards database [11] (https://www.genecards.org/) and Uniprot database constipation", a total of 2,842 constipation-related targets were collected.

\subsection{Screening and Network Construction of San Ren Tang for Constipation}

After matching the chemical component targets and constipation targets of Sanren decoction, 119 common targets of Sanren decoction and constipation, that is, the potential target of Sanren decoction in the treatment of constipation, are obtained, see Table 1.

\subsection{Chemical constituents of San Ren Tang-Target-Disease Network}

The chemical composition-target-disease network (PPI network Figure 2) was constructed using Cytoscape software [12] association function (Merge) with 118 nodes with 1,775 edges. Core nodes were screened by analyzing network topology parameters such as degree (degree) [13], proximity centrality (closeness centrality, CC) [14] and mediation centrality (betweenness centrality, BC) [14]. The larger the 3 parameter values, the closer the nodes are to the network the center of the network, the greater the role in network topology. Using association function cytohubba the PPI was MCC and the top ten targets were selected as AKT1, VEGFA, JUN, TP53, CASP3, IL6, TNF, PTGS2, MAPK1 and MMP9. The top 10 selected targets may be important targets for the treatment of constipation. 
Table 1. Potential therapeutic targets of Sanren Decoction for constipation

\begin{tabular}{|c|c|c|c|c|c|c|c|c|c|}
\hline DRD1 & NR3C2 & MAPK14 & BCL2L1 & TP53 & CCNB1 & CDK1 & NR1 I 2? & PPARD & IRF1 \\
\hline CHRM3 & ADRA2A & GSK3B & CDKN1A & NFKBIA & IFNG & HSPA5 & COL1A1 & CRP & ERBB3 \\
\hline CHRM1 & SLC6A2 & CCNA2 & CASP9 & TOP1 & IL4 & CYP3A4 & PTEN & CXCL10 & GSTM1 \\
\hline SCN5A & SLC6A3 & NOS2 & MMP9 & MDM2 & TOP2A & CYP1A2 & MPO & CHUK & APOD \\
\hline PTGS2 & AKR 1B1 & ESR2 & MAPK1 & MMP1 & GSTP1 & CAV1 & NFE2L2 & SPP1 & HTR3A \\
\hline RXRA & PLAU & DPP4 & IL10 & PCNA & MET & F3 & NQO1 & RUNX2 & HSPB 1 \\
\hline HTR2A & MAOB & CHEK1 & RB1 & ERBB2 & MMP3 & CYP1A1 & COL3A1 & RASSF1 & MYC \\
\hline CHRM2 & MAOA & NOS3 & CDK4 & HMOX1 & EGF & CCL2 & CXCL2 & $\mathrm{E} 2 \mathrm{~F} 1$ & PRKCA \\
\hline OPRM1 & GABRA3 & KCNMA 1 & TNF & ICAM1 & $\mathrm{ODC1}$ & SELE & NR1I3 & CTSD & BCL2 \\
\hline PGR & ESR1 & AKT 1 & JUN & MCL1 & CASP8 & PRKCB & CHEK2 & RASA1 & ADRA2C \\
\hline $\mathrm{ADH} 1 \mathrm{C}$ & PPARG & VEGFA & IL6 & BIRC5 & SOD1 & TGFB1 & CLDN4 & IGFBP3 & GABRA5 \\
\hline DRD2 & CCND1 & CASP3 & IL2 & STAT1 & MGAM & PPARA & IGF2 & MAP2 & \\
\hline
\end{tabular}

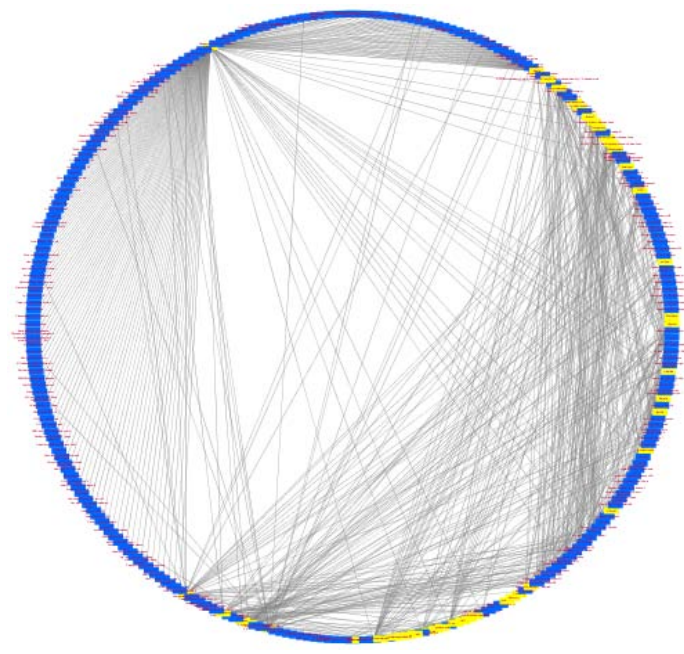

Figure 1A

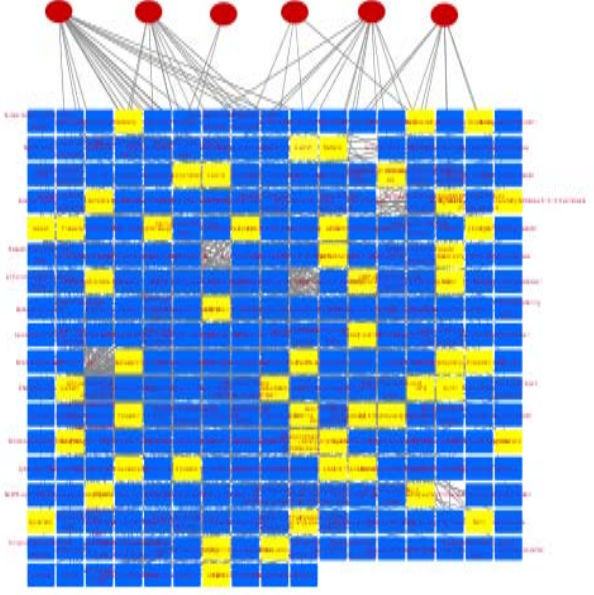

Figure 1B

Chemical constituents-action-target network of Sanjen Decoction Traditional Chinese Medicine of Sanren Decoction-Chemical Components-Target Map.

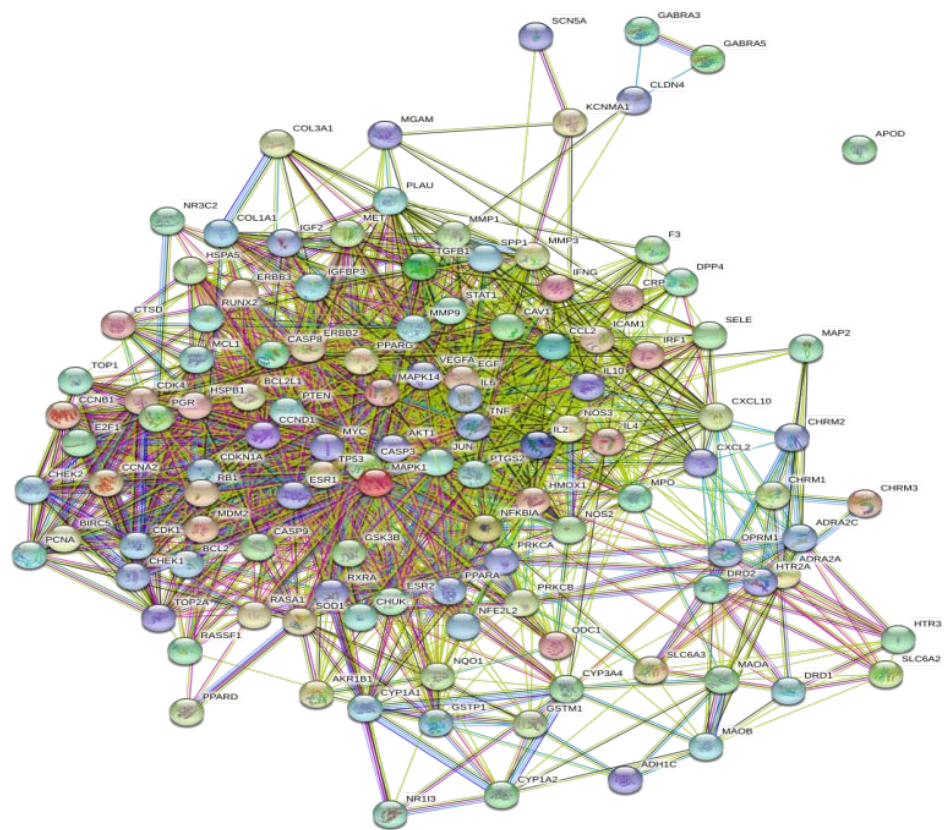

Figure 2. Chemical component-target-disease network diagram (PPI network diagram). 


\subsection{Analysis on Biological Process and Signal Pathway Enrichment of Sanren Decoction for Constipa- tion}

Gene function and signaling pathway analysis of potential targets of Sanren decoction for constipation using Clue GO. By P-value screening high-significant biological processes and signal pathways and making bubble map, the vertical axis indicates the biological process or pathway name, the horizontal axis indicates the enrichment factor, the bubble depth indicates the enrichment significance, and the bubble area size indicates the number of genes concentrated in this biological process or pathway. Relevant biological process and signal pathway information obtained by gene enrichment analysis. Analysis parameters were set by software default, network specificity was selected as medium, $\mathrm{P}<0.05$ show the results. Adoption The database explored the correlation between genes and diseases and analyzed the enrichment of related genes. The results of the first 20 biological processes (Figure 3) show that the main biological processes associated with the target are: panin-like protein ligase binding, kinase regulatory activity, cytokine receptor binding, ubiquitin protein ligase binding, protein kinase regulatory activity, phosphatase binding, cytokine activity, protease binding, neurotransmitter receptor activity, protein enzyme binding, steroid hormone receptor activity and RNA polymerase II transcription factor binding, nuclear receptor activity, transcription factor activity, direct ligand regulatory sequence specific activity sexual DNA binding, G protein-coupled amine receptor activity, ammonium ion binding, kinase activator activity, cyclin-dependent protein serine-threonine kinase regulator activity, histone kinase activity, catecholamine binding protein. It is suggested that Sanren decoction can play its role in treating constipation through multiple biological processes. According to the results of target pathway enrichment (Figure 4), the main pathways involved were PI3K-Akt signaling pathway (31 targets), hepatitis B pathway (25 targets), human cytomegalovirus infection pathway (24 targets), hepatitis C pathway (23 targets involvement), Kaposi’s sarcoma-associated herpesvirus infection pathway (23 targets involved), EB virus infection pathway (23 targets involved), proteoglycan pathway in cancer (22 targets involved), age-age signaling pathway in diabetic complications (22 targets involved), hepatocellular carcinoma pathway (22 targets involved), prostate cancer pathway (20 targets involved), fluid shear stress and atherosclerosis pathway (20 targets involved), cell senescence pathway (20 targets involved), IL17 signaling pathway (19 targets involved), TNF signaling pathway. Pathway No .19 and), small cell lung cancer pathway (18 targets involved), P53 signaling pathway (16 targets involved), etc. The results indicated that the targets of the active components of Sanren Decoction were distributed in different pathways and might play a therapeutic coordination role through multiple pathways.

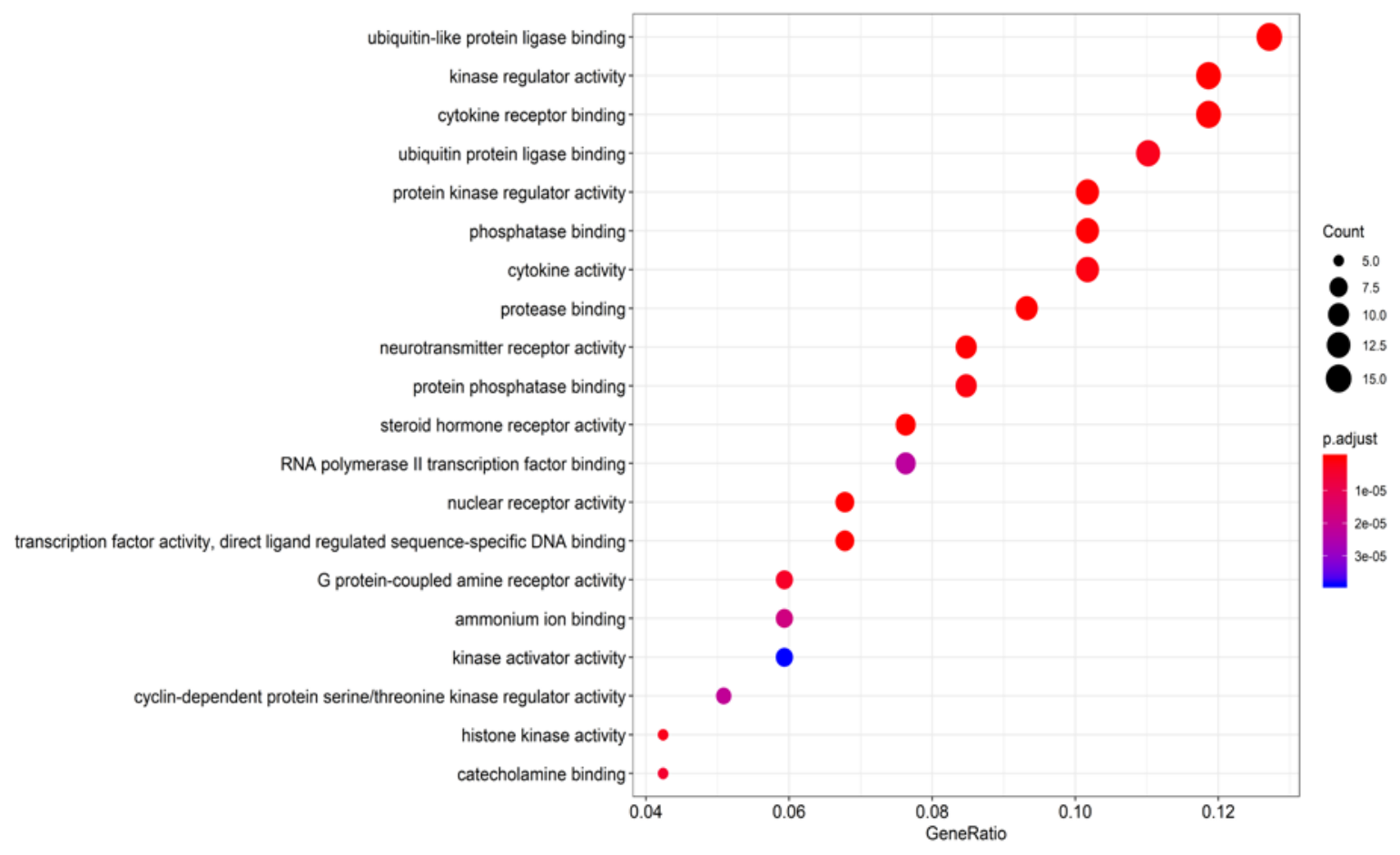

Figure 3. Bioprocess Enrichment Analysis of Sanren Decoction for Constipation Target. 


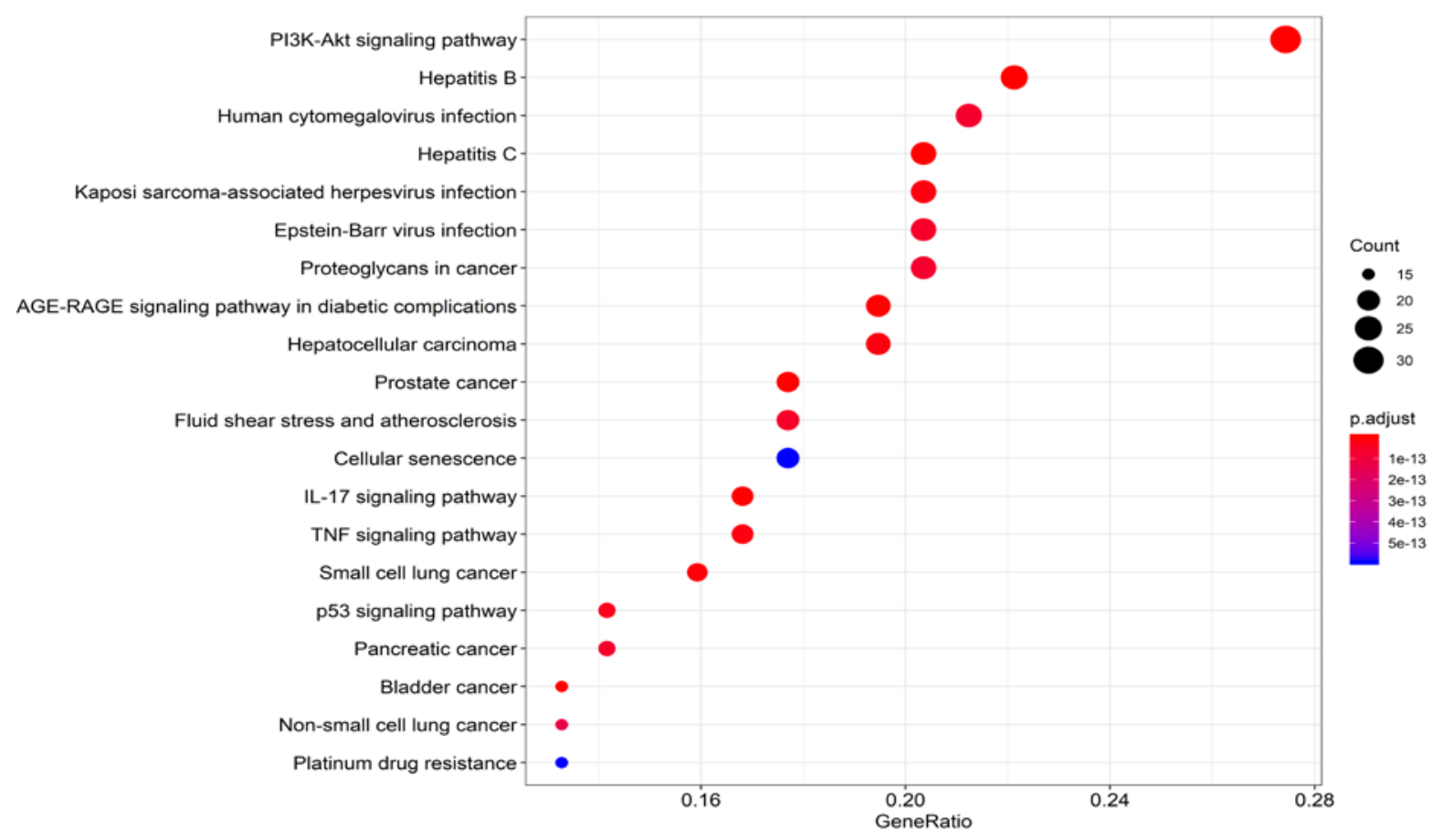

Figure 4. Enrichment Analysis of Pathway of Sanren Decoction for Treatment of Constipation Target.

\section{Discussion}

San Ren Tang is composed of 8 kinds of drugs, such as bitter almond, white cardamom, Coix seed, Magnolia officinalis, Pinellia ternata, Feiliu stone, Baitong grass and bamboo leaves. In this paper, based on big data and network pharmacology method, the treatment of constipation by Sanren decoction was systematically predicted and analyzed. There were 502 chemical constituents of San Ren Tang prescription from TCMSP and UniProt database, 219 corresponding targets, which were constructed The chemical constituents with high correlation with the action of Sanjen Decoction are soybean sterol, $\beta$-glutosterol, glutosterol, eicosadienoic acid, $\beta$-D-ribofuranoside, xanthine-9, estrone, diisooctyl succinate, quercetin, luteolin, glutenin $\alpha 1$, ne hesperidin, tetraploid Bct, which may be the main pharmacodynamic basis of Sanjen Decoction for constipation. Using association function cytohubba the PPI is MCC and the top ten targets are selected as AKT1, VEGFA, JUN, TP53, CASP3, IL6, TNF, PTGS2, MAPK1 and MMP9, may be important targets for the treatment of constipation with Sanren decoction. Some studies have shown that PTGS2 belongs to an inducible isoenzyme (COX-2) that can trigger intestinal motor dysfunction after obstruction [15]. Shi XZ [16] and other reports have shown that the use of COX-2 selective inhibitors can restore the contractility of intestinal smooth muscle strips and culture smooth muscle cells. Moreover, the degree of intestinal motor function decline after obstruction in COX-2 gene-deficient mice is significantly reduced. Does Sanren Decoction play a role in treating constipation by regulating COX-2 and other targets Use also requires experimental verification. The results of target-related signal pathway and biological process enrichment analysis showed that the main biological processes of target-related association were pantothenoid protein ligase binding, kinase regulatory activity, cytokine receptor binding, ubiquitin protein ligase binding, protein kinase regulator activity, phosphatase binding, cytokine activity, protease binding, neurotransmitter receptor activity, protein phosphatase binding, steroid hormone receptor activity and RNA polymerase II transcription factor binding, nuclear receptor activity, transcription factor activity, Specific binding DNA direct ligand regulatory sequences, G protein-coupled amine receptor activity, ammonium ion junction Synthesis, kinase activator activity, cyclin-dependent protein serine-threonine kinase regulator activity, histone kinase activity, catecholamine binding egg, etc. The main signaling pathways involved include: PI3K-Akt signaling pathway, hepatitis B pathway, human cytomegalovirus infection pathway, hepatitis C pathway, Kaposi's sarcoma-associated herpesvirus infection pathway, EB virus infection pathway, proteoglycan pathway in cancer, age-age signaling pathway for diabetic complications, hepatocellular carcinoma pathway, prostate cancer pathway, fluid shear stress and atherosclerosis pathway, cell senescence pathway, IL1. Signaling pathway, TNF signaling pathway, small cell lung 
cancer pathway, P53 signaling pathway, etc., among them, the signal pathway predicted in this paper for the treatment of constipation by Sanren decoction is more related to the PI3K-Akt signaling pathway (phosphatidylinositol-3 kinase/serine-threonine protein kinase signaling pathway). A number of studies have shown that PI3K-Akt signaling pathway is an important signal transduction pathway in cells, which plays an important role in inhibiting apoptosis and promoting cell proliferation. PI3K is a cytosolic phosphatidylinositol kinase that is activated and forms PIP3, PIP3 as a second messenger Over-transmission signals have biological effects, which include Akt phosphorylation. Phosphorylated Akt can be involved in promoting cell proliferation, metabolism, inhibiting apoptosis, dilating blood vessels, relaxing smooth muscle and inhibiting intestinal peristalsis [17-22]. Some studies have shown that PI3K/AKT signaling pathway can improve mitochondrial function by regulating NO, regulate apoptosis and regulate intestinal function through activation, and can regulate NO and guanylyl cyclase, and relax intestinal smooth muscle to inhibit intestinal peristalsis, slow transport constipation [23-25]. Tip PI3K-A kt signaling pathway may be one of the important mechanisms of Sanren decoction to interfere with constipation, but it is still reported in the literature that Sanren decoction interferes with constipation through PI3K-Akt signaling pathway. The results of KEGG pathway analysis can be seen that Sanren decoction can also treat constipation by regulating hepatitis B pathway, human cytomegalovirus infection pathway, hepatitis C pathway, Kaposi's sarcoma-associated herpesvirus infection pathway, EB virus infection pathway and so on. Among the predicted targets and signaling pathways of Sanren Decoction for the treatment of constipation, PI3K-Akt signaling pathway has been reported to be involved in constipation mechanism. In addition, other targets, signaling pathways have not been reported, which is the first time in this paper, which provides a reference direction for future research. Based on data analysis and network pharmacology, this study discussed the mechanism of Sanren Decoction in treating constipation, explained the active components of Sanren Decoction in treating constipation and predicted the possible molecular mechanism. But the method of studying Chinese medicine compound to treat diseases based on network pharmacology has There are some limitations, many drugs and diseases in the database search process cannot find the corresponding active components and targets, such as: San Ren Tang talc, bamboo leaves of the active components are 0 , so it is impossible to find their corresponding targets. These two drugs may also play a role in the treatment of constipation by San Ren Tang. At the same time, this study cannot be clear about the metabolic process of Sanren decoction and its metabolites on the body. Therefore, this study has some limitations, it is necessary to do further research on the treatment of constipation Sanren decoction and experimental verification of the theoretical results of this study.

\section{References}

[1] Jasper Pannemans, Karen Van den Houte, Benjamin Fischler, Hubert Piessevaux, Florencia Carbone, Jan Tack. (2020). Prevalence and impact of selfreported painful and non-painful constipation in the general popu lation [J]. Neurogastroenterology \& Motility, 2020, 32(4).

[2] Ye Mengyi, Niu Yang. (2017). Clinical Application of Sanren Decoction Bright Chinese Medicine, 2017, 32(06): 887-890.

[3] Wang Chengchuan, Liu Qiang, Peng Qifeng, Wang Yirui, Yan translated Xi, he Ping. (2018). An Analysis of Professor He Ping's Experience in Treating Dehumidification and Heat Stagnation Constipation Based on Syndrome Differentiation of Sanjiao [J]. Sichuan TCM, 2018, 36(06): 7-9.

[4] Dou Xibin, Tang Hanqing. (2019). Application of Sanren Decoction [J]. Journal of Guangxi University of Traditional Chinese Medicine, 2019, 22(02): 38-40.

[5] Guo Dongfang, Luo Wenzhao, Zhang Weidong, Sun Yuxin. (2008). Application of San Ren Tang by Prof. Sun Yuxin [J]. New Chinese Medicine, 2008(05): 115.

[6] Duan Qi, Jiang An, Li Zhipeng, Yang Dong, He Ping. (2007). Add or subtract Sanren Decoction to treat 35 cases of dampness and heat accumulation of spleen constipation [J]. Colorectal Anal Surgery, 2007(04): 245-246.

[7] Zhang Huamin, Liu Sihong, Gao Hongjie, Gong Gupta Lei. (2019). Advances in Pharmacological Methods of Compound Traditional Chinese Medicine Network [J]. Evaluation and Analysis of Drug Use in Chinese Hospitals, 2019, 19(10): $1270-1276$.

[8] Jinlong Ru, Peng Li, Jinan Wang, Wei Zhou, Bohui Li, Chao Huang, Pidong Li, Zihu Guo, Weiyang Tao, Yinfeng Yang, Xue $\mathrm{Xu}$, Yan Li, Yonghua Wang, Ling Yang. (2014). TCMSP: a database of systems pharmacology for drug disc overy from herbal medicines [J]. Springer International Publishing, 2014, 6(1).

[9] Liu Zhongyang, Guo Feifei, Wang Yong, Li Chun, Zhang Xinlei, Li Honglei, Diao Lihong, Gu Jiangyong, Wang Wei, Li Dong, He Fuchu. (2016). BATMAN-TCM: a Bioinformatics Analysis Tool for Molecular mechanism of Traditional Chinese Medicine [J]. Pubmed, 2016, 6.

[10] Luo Jingchu. (2019). UniProt Protein Database [J]. Bioinformatics, 2019, 17(03): 131-144. 
[11] Fichilevich Simon, Noodles Ron, Rapa Portnoya, Hadar Red, Prasquez Inbar, Inestantztipi, Rosennaomi, Cohen Asher, Twik Michal, Saffron Marilyn, Lancet Dolon, Kendana. (2017). GeneHancer: genome-wide integration on of enhancers and target genes in GeneCards [J]. Pubmed, 2017.

[12] Holmås Stian, Puig Rafel Riudavets, Acencio Marcio Luis, Mironov Vladimir, Kuiper Martin. (2019). The Cytoscape BioGateway App: explorative network building from the BioGateway triple store [J]. Pubmed, 2019.

[13] Amit Kumar Subudhi, Pon Arunachalam Boopathi, Isha Pandey, Ramandeep Kaur, Sheetal Middha, Jyoti Acharya, Sanjay K. Kochar, Dhanpat K. Kochar, Ashis Das. (2015). Disease specific modules and hub genes for interv ention strtegies: A co-expression network based approach for Plasmodium falciparum clinical isolates [J]. Infection, Genetics and Evolution, 2015, 35.

[14] Yu Tang, Min Li, Jianxin Wang,Yi Pan, Fang-Xiang Wu. (2015). CytoNCA: A cytoscape plugin for centrality analysis and evaluation of protein interaction networks [J]. BioSystems, 2015, 127.

[15] Zong Yang, Sun Mingming, Yue Yinzi, Yan Shuai. (2018). A study on the mechanism of Atractylodes macrocephala-Fructus Aurantii in the treatment of chronic transit constipation based on network pharmacology [J]. China Pharmacy, 2018, 29(13): 1798-1802.

[16] Shi XZ, Lin YM, Powell DW, et al. (2011). Pathophysiology of motility dysfunction in bowel obstruction: role of stretch-induced COX-2 [J]. Am J Physiol Gastrointest Liver Physiol, 2011, 300(1): G99-G108.

[17] Li Jia. (2016). Role of PI3K/AKT/GSK3-b signal transduction pathway in salidroside protection of myocardial ischemia-reperfusion injury in rats [J]. Journal of Cardiovascular and Cerebrovascular Diseases, 2016, 14(22): 2621-2624.

[18] Shahad W. Kattan, Mohamed S. Nafie, Gamal A. Elmgeed, Walla Alelwani, Muhammad Badar, Mohamed A. Tantawy. (2020). Molecular docking, anti-proliferative activity and induction of apoptosis in human liver cance $\mathrm{r}$ cells treated with androstane derivatives: Implication of PI3K/AKT/mTOR pathway [J]. Elsevier Ltd, 2020, 198.

[19] Zhang Huamin, Liu Sihong, Gao Hongjie, Gong Gupta Lei. (2019). Advances in Pharmacological Methods of Compound Traditional Chinese Medicine Network [J]. Evaluation and Analysis of Drug Use in Chinese Hospitals, 2019, 19(10): 1270-1276.

[20] Liu Hong, Zhang Lingyan, Xue Yang. (2019). Experimental study of Xuefu Zhuyu decoction to relieve myocardial ischemia-reperfusion injury in rats through PI3K/Akt pathway [J]. Pharmacology and Clinical Medicine, 2019, 35(05): 11-15.

[21] Li Lei, Gao Yizhao, Ma Lianping, Miao Cheng, Yang Hui. (2019). Asiaticoside attenuates brain tissue damage and induces angiogenesis in focal cerebral infarction rats by activating PI3K/Akt signaling pathway [J]. Chinese Journal of Immunology, 2019, 35(17): 2068-2078.

[22] Zhu Jing, Song Yue. (2019). Mechanism of low dose LBH589 inducing apoptosis of epithelial ovarian cancer cells through PI3K/AKT pathway [J]. Journal of Bengbu Medical College, 2019, 44(06): 708-711.

[23] Deng Heng. (2018). Study on c-kie mRNA of colonic tissue and PI3K/AKT/eNOS signaling pathway in rats with slow transit constipation [D]. Anhui University of Traditional Chinese Medicine.

[24] Li Minhao, Zhang Guozhi, Yuan Mei, Li Shuguang, Chen Junmao. (2015). The expression changes and significance of PI3K/AKT/eNOS signaling pathway related markers in gastrointestinal tissues of slow-transmission constipation rats [J]. Shandong Medicine, 2015, 55(19): 27-29.

[25] Li Minhao. (2015). Role of PI3K/AKT/eNOS signaling pathway in chronic transport constipation in rats [D]. North China University of Technology. 